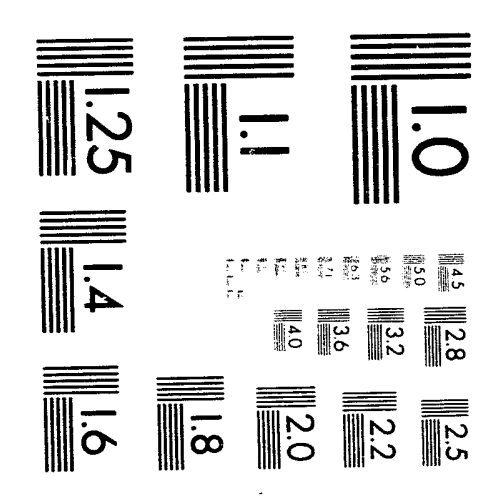



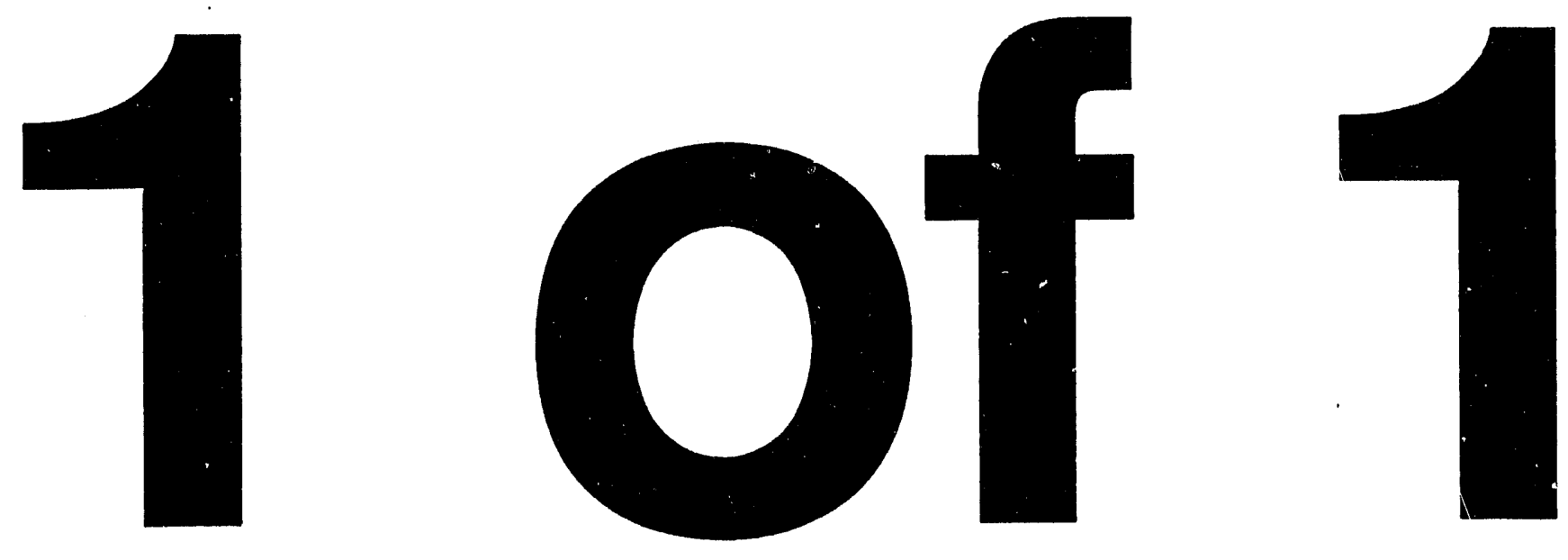


\title{
A Unified Monte Carlo Interpretation of Particle Simulations and Applications to Nonneutral Plasmas
}

\author{
A. Y. Aydemir \\ Institute for Fusion Studies \\ The University of Texas at Austin \\ Austin, Texas 78712
}

\begin{abstract}
Using a "Monte Carlo interpretation" of particle simulations, a general description of low-noise techniques is developed in terms well-known Monte Carlo variance reduction methods. Some of these techniques then are applied to linear and nonlinear studies of pure electron plasmas in cylindrical geometry, with emphasis on the generation and nonlinear evolution of electron vortices. Long-lived $l=1$ and $l=2$ vortices, and others produced by unstable diocotron modes in hollow profiles, are studied. It is shown that low-noise techniques make it possible to follow the linear evolution and saturation of even the very weakly unstable resonant diocotron modes.
\end{abstract}

PACS No's: $52.25 . \mathrm{Wz}, 52.65 .+z, 52.55 . \mathrm{Mg}$ 


\section{Introduction}

Particle simulations in which the plasma is treated as a collection of a large number of "computational particles," interacting with each other through self-consistently generated fields, is an important tool in computational and theoretical studies of plasmas. Over many decades of research, a vast number of techniques have been developed in this field, many of which can now be found in classic texts and monographs on the subject. $1,2,3$ In very broad terms, this approach to the study of plasmas involves two distinct steps: calculation of the self-consistent fields generated by a given distribution of computational particles in a multi-dimensional phase space, and following the particle orbits in these fields. Although solution of the dynamical equations in the second step of this process introduces some error

and noise, the "noise in particle simulations" is predominantly associated with the first step, where low-order moments of the distribution function are calculated to find the source terms for Poisson's or Ampére's equations. This noise is essentially the error introduced when evaluating the moments using a relatively small number of points in phase space, determined by computational particle positions.

Mathematically, calculation of various moments of the distribution function in particle simulations is equivalent to a Monte Carlo evaluation of integrals in a large dimensional phase space $^{4}$; thus, the particle simulation technique lends itself to a "Monte Carlo interpretation," where computational particles are regarded as Lagrangian markers which carry with them some information about the distribution function, and which are used in Monte Carlo integral evaluations to obtain the necessary moments. ${ }^{5}$ This point of view shifts the emphasis from particles to the observable quantities, such as the particle or current density, obtained by taking moments of the distribution function. It also makes transparent the source of noise and points to various methods available for reducing it. 
Since the noise in the simulations and the integral evaluations are intimately connected, in order to understand various noise reduction methods, we devote the first part of the article (Sec. II) to a general discussion of particle simulations, and various Monte Carlo techniques used in integral evaluations in large dimensional spaces. In the second part, some of these techniques are applied to a concrete physics problem, where we examine the linear and nonlinear stability of diocotron modes and long-lived vortices generated by them, in pure electron plasma columns in cylindrical geometry.

\section{Monte Carlo interpretation}

In this section we examine in some detail the Monte Carlo interpretation of particle simulations and discuss some of the classical techniques available for noise reduction in Monte Carlo evaluation of integrals. We begin with an elementary discussion of a generalized kinetic equation associated with a given set of equations of motion.

\section{A. The kinetic equation}

Let the nonlinear equations of motion for particles in an $M$-dimensional phase space be represented by

$$
\dot{Z}^{i}=\xi^{i}\left(Z^{1}, Z^{2}, Z^{3}, \ldots, Z^{M}, t\right)
$$

where $M$ is the dimension of the phase space, and $Z^{i}, i=1,2, \ldots, M$ are generalized coordinates.

Also let $f=f(\mathbf{Z}, t)$ be a distribution function such that $f(\mathbf{Z}, t) d \Gamma$ is the number of particles in the volume element $d \Gamma=\mathcal{J} d Z^{1} d Z^{2} \cdots d Z^{M}$, where $\mathcal{J}$ is the Jacobian of the transformation from $\mathbf{Z} \equiv\left(Z^{1}, Z^{2}, \ldots, Z^{M}\right)$ to an appropriate Cartesian coordinate system. Ignoring short-range interactions (collisions), conservation of particles in the volume element $d \Gamma$ implies

$$
\frac{\partial}{\partial t}(\mathcal{J} f)+\frac{\partial}{\partial Z^{i}}\left(\dot{Z}^{\prime} \mathcal{J} f\right)=0
$$


where $\dot{\mathrm{Z}}$ is given by Eq. (1). In addition, if the equations of motion preserve the phasespace volume element $d \Gamma$, or equivalently, if the phase space volume $\int \mathcal{J} d \mathbf{Z}$ is an invariant integral ${ }^{6,7}$ of Eq. (1), we can easily obtain the following statement of the Liouville theorem,

$$
\frac{\partial}{\partial t} \mathcal{J}+\frac{\partial}{\partial Z^{i}}\left(\dot{Z}^{i} \mathcal{J}\right)=0 .
$$

In this case, the kinetic equation simplifies to its more usual form

$$
\frac{d f}{d t}=\frac{\partial f}{\partial t}+\dot{Z}^{i} \frac{\partial f}{\partial Z^{i}}=0 .
$$

During the rest of our discussion, we will assume that the Liouville theorem, Eq. (3), holds and use the simpler form of the kinetic equation in (4).

Following the particle orbits using Eq. (1), as it is typically done in particle simulations, or solving for the distribution function $f$ using Eq. (2), or (4), along the characteristics defined by Eq. (1), is of course completely equivalent. Both techniques, however, require calculation of various moments of the distribution function; therefore, we now turn to an examination of Monte Carlo methods in evaluation of such integrals.

\section{B. Monte Carlo evaluation of integrals}

In the context of our discussion, the term Monte Carlo simply refers to estimation of multidimensional integrals using statistical sampling techniques. In general, particular form of the integrand and how it is sampled in the volume of interest determine the accuracy of the estimates. ${ }^{4}$ Over many decades of research since the 1950's, the Monte Carlo community has developed a number of techniques that try to minimize the error in the estimates and increase the efficiency of the calculations. Below we review some of these techniques and their application in particle simulations.

Our discussion will be in terms of a general integral of the form

$$
I(A) \equiv \int_{V} A(\mathbf{Z}) f(\mathbf{Z}) d \Gamma
$$


where the volume element is $d \Gamma=\mathcal{J} d Z^{1} d Z^{2} \cdots d Z^{M}$, and $A(\mathbf{Z})$ is a general function of the phase space coordinates $\mathrm{Z}$. For example, $I(A)$ would be the number density in configuration space if $A=1$, and the integral is over the velocity space.

If we treat $\mathbf{Z}$ as a continuous random variable with a probability density $p(\mathbf{Z})$ in the phase space volume $V$ such that

$$
\int_{V} p(\mathbf{Z}) d \Gamma=1
$$

then we can rewrite the integral for $I(A)$ to obtain

$$
I(A)=\langle g\rangle \equiv \mathcal{E}(g(\mathbf{Z}))=\int_{V}\left\{\frac{A(\mathbf{Z}) f(\mathbf{Z})}{p(\mathbf{Z})}\right\} p(\mathbf{Z}) d \Gamma
$$

where $\mathcal{E}(g)$ is the expected value of the random variable $g \equiv A(\mathbf{Z}) f(\mathbf{Z}) / p(\mathbf{Z})$. Similarly, we define the variance of $g$ by

$$
\mathcal{V}(g)=\sigma^{2}=\int_{V}(g-\langle g\rangle)^{2} p(\mathbf{Z}) d \Gamma
$$

Using an independent random sample $\left(\mathbf{Z}_{1}, \mathbf{Z}_{2}, \ldots, \mathbf{Z}_{N}\right)$ from the random variable $\mathbf{Z}$, we can also define a sample mean $\bar{g}$ as

$$
\bar{g}=\frac{1}{N} \sum_{j=1}^{N} g\left(\mathbf{Z}_{j}\right) .
$$

Note that for the sake of economy of notation, we let $\mathbf{Z}_{j}$ refer both to a random variable in the sample, and a particular value the variable may assume. It is straightforward to show that $\bar{g}$ is an unbiased $(\mathcal{E}(\bar{g})-\langle g\rangle=0)$, and consistent $\left(\mathcal{V}(\bar{g})=\sigma^{2} / N \rightarrow 0\right.$ as $\left.N \rightarrow \infty\right)$ estimate $^{8}$ for $\langle g\rangle \equiv \mathcal{E}(g)=I(A)$, with a standard error $\epsilon \simeq \sigma / \sqrt{N}$, where $\sigma$ is the standard deviation defined in Eq. (7).

Thus, we conclude that a valid Monte Carlo estimate for the general moment integral $I(A)$ is given by

$$
I(A)=\frac{1}{V} \sum_{j=1}^{N} \frac{A\left(\mathbf{Z}_{j}\right) f\left(\mathbf{Z}_{\jmath}\right)}{p\left(\mathbf{Z}_{\jmath}\right)}+\epsilon, \quad \epsilon \simeq \frac{\sigma}{\sqrt{N}}
$$


To be able to make use of this result in particle simulations, we note that we can associate the values the random sample $\left(\mathbf{Z}_{1}, \mathbf{Z}_{2}, \ldots, \mathbf{Z}_{N}\right)$ can assume with computational particle (or Lagrangian marker) positions in phase space. Then moving the markers along the orbits defined by Eq. (1) while letting them carry some information about the distribution function $f$ will enable us to calculate the time evolution of $f$ and its moments. The required information (or weight) assigned to each Lagrangian marker can be determined by rewriting Eq. (9) in terms of a Klimontovich density (we ignore the error term for the moment):

$$
I(A)=\int_{V} A(\mathbf{Z})\left[\frac{1}{\mathcal{J}(\mathbf{Z})} \sum_{j}^{N} \frac{1}{N} \frac{f\left(\mathbf{Z}_{j}\right)}{p\left(\mathbf{Z}_{j}\right)} \delta\left(\mathbf{Z}-\mathbf{Z}_{j}\right)\right] \mathcal{J} d \mathbf{Z} .
$$

We associate the term in square brackets, with the Klimontovich density $f_{K}(\mathbf{Z})$ for the Lagrangian markers:

$$
f_{K}(\mathbf{Z}) \equiv \frac{1}{\mathcal{J}(\mathbf{Z})} \sum_{j=1}^{N} w_{j} \delta\left(\mathbf{Z}-\mathbf{Z}_{j}\right)
$$

where the weight $w$; is given by

$$
w_{j} \equiv \frac{1}{N} \frac{f\left(\mathbf{Z}_{j}\right)}{p\left(\mathbf{Z}_{j}\right)} .
$$

Note that by construction, we have, for any volume element $\Omega$ in $V$,

$$
\int_{\Omega} f(\mathbf{Z}) d \Gamma=\int_{\Omega} f_{K}(\mathbf{Z}) d \Gamma+\Omega=\sum_{\mathbf{Z}, \in \Omega} w_{\jmath}+\epsilon_{\Omega}=N_{\mathfrak{s}}(\Omega)+\epsilon_{\Omega}
$$

where $V_{s}(\Omega)$ is the number of physical particles in $\Omega$, and $\epsilon_{\Omega} \sim \sigma / \sqrt{N}$ characterizes the error in the Monte Carlo estimate for $N_{s}(\Omega)$. We assume at this point that for $\Omega=V$, the integral of $f_{K}$ gives the exact answer, without an error term:

$$
\int_{V} f_{K}(\mathbf{Z}) d \Gamma=\sum_{j=1}^{N} w_{j}=N_{s}
$$

where $N_{s}$ is the total number of physical particles in the system $\left(N_{0} \gg N\right)$. In general, the exact equality will not hold, as it will be discussed in more detail in Sec. II.C.

Summarizing our results up to his point, ve have the following: 
- $f(Z)$ is the physical particle distribution function.

- $p(\mathbf{Z})$ is a probability density for the random variable $\mathbf{Z}$ and the random sample $\left(\mathbf{Z}_{1}, \mathbf{Z}_{2}, \ldots, \mathbf{Z}_{N}\right)$. These $N$ random points in the $M$-dimensional phase space also specify positions of the computational particles (or Lagrangian markers).

- $f_{K}$ is a Klimontovich density for the Lagrangian markers defined such that moments of $f$ can be estimated using $f_{K}$ as follows:

$$
\int_{\Omega} A(\mathbf{Z}) f(\mathbf{Z}) d \Gamma \simeq \int_{\Omega} A(\mathbf{Z}) f_{K}(\mathbf{Z}) d \Gamma=\sum_{\mathbf{Z}, \in \Omega} A\left(\mathbf{Z}_{j}\right) w_{j},
$$

for any volume element $\Omega$ in $V$. An estimate of the error in the Monte Carlo approximation, usually associated with "noise" in particle simulations, is given by $\epsilon \simeq \sigma / \sqrt{N}$, where $\sigma$ is defined in Eq. (7).

- Finally, dynamics of the Lagrangian markers is described by the physical equations of motion (Eq. 1):

$$
\frac{d \mathbf{Z}_{j}}{d t}=\boldsymbol{\xi}_{j}\left(\mathbf{Z}_{1}, \mathbf{Z}_{2}, \ldots, \mathbf{Z}_{N}, t\right) .
$$

Equations (11) and (12) for the Klimontovich density and the particle weights, and the equations of motion in (1), together with those equations that relate the source terms obtained from moments of $f_{K}$ to the fields (not specified here), comprise a closed set of equations that can be used in a generalized, self-consistent, particle simulation algorithm. Below we examine a number of techniques developed in the Monte Carlo community, involving specific choices for $p(\mathbf{Z})$, or a rearrangement of the integrand $A(\mathbf{Z}) f(\mathbf{Z})$ in Eq. (5), that help reduce the error (noise) in the estimates for $I(A)$.

\section{Simple (crude) Monte Carlo}

In the simplest Monte Carlo method, a uniform probability density $p(Z)$ is chosen for the random sample $\left(\mathbf{Z}_{1}, \mathbf{Z}_{2}, \ldots, \mathbf{Z}_{N}\right)$, i.e., Lagrangian markers are uniformly distributed in phase 
space. Assuming a finite phase space volume $V$ (in practice, $V$ is always finite since a cutoff is introduced in velocity space integrals), we have

$$
p(\mathbf{Z})=\frac{1}{V}, \quad w_{j}=\frac{V}{N} f\left(\mathbf{Z}_{j}\right), \quad \text { and } g=V A(\mathbf{Z}) f(\mathbf{Z}) .
$$

Note that $V / N$ represents a marker's share of the phase space and is guaranteed to be constant by the Liouville theorem along the particle orbit. Similarly, $f_{j}$ is constant, implying that the particle weight $w_{j}$ is a constant of motion. However, $w_{j}$ is different for each particle; in practice, a simulation would have to keep track of this information for all the particles, which is one of the drawbacks of this method. A sec ad problem with this method is that it is not very efficient: phase space is covered uniformly with markers, although certain portions of the phase space may be more important than others; for example, what happens at the tails of the distribution function $f$ may not be relevant to the problem at hand.

A more serious problem with the simple Monte Carlo method is that it is not a particularly "low-noise" technique. Since the function $g$ of Eq. (6) is directly proportional to the distribution function $f$, which in general varies by many orders of magnitude over the phase space, the difference between $g$ and its expected value $\langle g\rangle$ can be very large, leading to a large variance $\mathcal{V}(g)$ (Eq. (7)). Thus, the error in the crude Monte Carlo estimates of moment integrals and the associated "noise" tend to be large compared to other methods discussed below. For these reasons, the simple Monte Carlo technique is not widely used in particle simulations by itself, although it has been used in conjunction with other methods.

\section{Importance sampling}

In this method, origins of which can be traced back to the Metropolis et al. algorithm, ${ }^{9}$ those portions of phase space that are "important" are sampled more frequently than others by choosing a nonuniform marker probability density $p(\mathbf{Z})$ proportional to the particle 
distribution function:

$$
p(\mathbf{Z})=\frac{1}{N_{s}} f(\mathbf{Z}), \quad w_{j}=\frac{N_{s}}{N}, \quad \text { and } \quad g=N_{s} A(\mathbf{Z})
$$

Recall that $N_{s}$ is the total number of physical particles in the system, and $N$ is the number of Lagrangian markers. Since all markers now have the same weight $N_{s} / N$, the information storage requirement is reduced; sometimes the Lagrangian markers are referred to as "macro particles," each representing $N_{s} / N$ physical particles. More importantly, all the variation in $g$ now comes from the function $A(Z)$, since $f / p=$ constant. Thus, in general, variance $\mathcal{V}(g)$ is reduced considerably, making importance sampling a more quiet technique than the simple Monte Carlo. For these reasons, and also since loading computational particles with the same distribution function as that of the problem being investigated is an intuitively obvious approach, this terhnique has been the mainstay of all classic particle simulation algorithms.

Further reduction in noise is possible by rearranging the integrand in Eq. (5) for $I(A)$.

\section{Control variates- $\delta f$}

Simple Monte Carlo and importance sampling techniques discussed above involve specific choices for the marker probability density $p(\mathbf{Z})$. Control variates method is another intuitively obvious approach that tries to reduce the variance in $I(A)$ by replacing as much of the Monte Carlo estimate as possible by analytic or numerical calculations that are more accurate.

Assume that there exists a function $f_{0}$, formally called the control variate, ${ }^{4,10}$ such that:

- moments of $f_{0}$ can be found easily, preferably analytically, and

- at all times, the physical distribution function $f(\mathbf{Z})$ remains close to $f_{0}(\mathbf{Z})$ in the sense:

$$
\left\|f-f_{0}\right\| /\|f\| \ll 1 \text {, using some arbitrary norm . }
$$


Then the error in the estimate for $I(A)$, which we identify with "noise" in particle simulations, can be reduced by rewriting the integral in the form

$$
I(A)=\int_{V} A(\mathbf{Z}) f_{0}(\mathbf{Z}) d \Gamma+\int_{V} A(\mathbf{Z})\left\{f(\mathbf{Z})-f_{0}(\mathbf{Z})\right\} d \Gamma
$$

and applying a Monte Carlo technique only to the second integral. As long as $\delta f \equiv f-f_{0}$ is small, we would expect the error in our Monte Carlo estimate of $I(A)$ to be small also. This classic technique of reducing the variance in the Monte Carlo estimate of an integral has been rediscovered ${ }^{11,12}$ and expanded upun ${ }^{13,14}$ independently by workers in the fusion community; it is now variously referred to as the " $\delta f$ ", or the "linear/nonlinear characteristic" method.

Proceeding as before, we now obtain the following Monte Carlo estimate for $I(A)$ :

$$
I(A)=I_{0}(A)+\frac{1}{N} \sum_{j=1}^{N} \frac{A\left(\mathbf{Z}_{j}\right) \delta f\left(\mathbf{Z}_{j}\right)}{p\left(\mathbf{Z}_{j}\right)}+\epsilon_{\delta f}, \quad \epsilon_{\delta f} \simeq \frac{\sigma_{\delta f}}{\sqrt{N}}
$$

where

$$
I_{0}(A) \equiv \int_{V} A(\mathbf{Z}) f_{0}(\mathbf{Z}) d \Gamma
$$

is the contribution from $f_{0}$, obtained analytically. The error (noise) now comes entirely from the small $\delta f$ integral; the variance $\sigma_{\delta f}^{2}$ is given by

$$
\mathcal{V}(\delta g)=\sigma_{\delta f}^{2}=\int_{V}(\delta g-\langle\delta g\rangle)^{2} p(\mathbf{Z}) d \Gamma,
$$

where $\delta g \equiv A(\mathbf{Z}) \delta f(\mathbf{Z}) / p(\mathbf{Z})$, and $\langle\delta g\rangle \equiv \mathcal{E}(\delta g)$, the expected value of $\delta g$. Comparing the error terms in Eq. (9) and Eq. (17), we see that the control variate technique achieves a reduction in noise of the order $\delta f / f$, for the same number of sample points (Lagrangian markers).

As in Eq. (11), we can associate a discrete distribution function $\delta f_{K}(\mathbf{Z})$ (a Klimontovich density) with the Lagrangian markers, where the particle weights are now given by

$$
w,(t)=\frac{1}{N} \frac{\delta f\left(\mathbf{Z}_{j}\right)}{p\left(\mathbf{Z}_{\jmath}\right)} .
$$


In terms of $\delta f_{K}(\mathbf{Z})$, the moment integral now becomes

$$
I(A) \simeq I_{0}(A)+\sum_{j=1}^{N} A\left(\mathbf{Z}_{j}\right) w_{j} .
$$

A complication introduced by the control variate method is that the particle weights are no longer constants of motion, since they depend on $\delta f$, not the full distribution function $f$. Using $\delta f=f-f_{0}$, we obtain

$$
\frac{d \delta f}{d t}=-\frac{d f_{0}}{d t}
$$

which simply leads to the following time-evolution equation for $w_{j}$ :

$$
\frac{d w_{j}}{d t}=-\frac{1}{N}\left[\frac{1}{p(\mathbf{Z})} \frac{d f_{0}}{d t}\right]_{\mathbf{Z}=\mathbf{Z},}
$$

So far in this section we have not specified the probability density $p(Z)$ for marker locations in phase space. Adopting the simple Monte Carlo approach discussed above, we may choose a uniform density, $p(Z)=1 / V$. This was evidently the method used by Kotschenreuther ${ }^{12}$ in the first successful application of the technique to particle simulations. However, it is more efficient to use the importance sampling method and let $p(\mathbf{Z})=f(\mathbf{Z}) / N_{s}$, as it has been also pointed out by others. ${ }^{13,14}$ In this case we get

$$
w_{j}(t)=\frac{N_{s}}{N} \frac{\delta f\left(\mathbf{Z}_{j}\right)}{f\left(\mathbf{Z}_{j}\right)},
$$

and

$$
\frac{d w_{j}}{d t}=-\frac{N_{s}}{N}\left(1-\frac{N}{N_{s}} w_{j}\right)\left[\frac{1}{f_{0}} \frac{d f_{0}}{d t}\right]_{\mathbf{Z}=\mathbf{Z},},
$$

where we recall $N$, is the total number of physical particles in the system, and $N$ is the number of Lagrangian markers. It is convenient to redefine $w_{j} \equiv \delta f_{j} / f_{j}$ and introduce the factor $N_{s} / N$ explicitly into the moment calculations, in which case we get

$$
\frac{d w_{j}}{d t}=-\left(1-w_{j}\right)\left[\frac{1}{f_{0}} \frac{d f_{0}}{d t}\right]_{\mathbf{Z}=\mathbf{Z},},
$$


which has been also obtained by Parker and Lee ${ }^{14}$ following a somewhat different, less transparent, approach. Note that under normal circumstances, it is not necessary to solve Eq. (22) in order to determine $w_{j}$; for obvious reasons, $w_{j}=\delta f_{j} / f_{j}$ is the solution to Eq. (22).

As a final point on the subject, we note that low-noise is not synonymous with high accuracy. As it has been pointed out earlier, the term "noise" in particle simulations refers to the overall error in the moment integral for $I(A)$. The control variate- $\delta f$ technique reduces noise by reducing the size of the Monte Carlo contribution to $I(A)$. But this method also concentrates all the relevant physics into this small integral of $\delta f$ and its time evolution; thus, the accuracy of the method crucially depends on accurate evaluation of the moments of $\delta f$. In other words, we have two complimentary requirements:

- Low noise is accomplished by ensuring $\left\|f-f_{0}\right\| /\|f\| \ll 1$.

- Accuracy, however, requires that the relative error in $\delta I(A)$ be small: $\left\|\epsilon_{\delta f} / \delta I(A)\right\| \ll 1$, where $\delta I(A) \equiv I(A)-I_{0}(A)$, and $\epsilon_{\delta f}$ is defined in Eqs. (17) and $(18)$.

The first objective can be met with a well-chosen control variate $f_{0}$ and a small $N$. The second one may still require a large number of markers, since $\epsilon_{\delta f} / \delta I(A) \sim 1 / \sqrt{N}$, i.e. it is independent of the size of $\delta f$ and only depends on $N$.

\section{Particle conservation}

Energy or momentum conservation cannot be discussed without specializing to a particular system of equations of motion; however, particle conservation properties of various schemes follow directly from the discussion on general moment integrals, in particular Eqs. (9), and (1i). Letting $A=1$ in Eq. (9), we obtain

$$
V_{\mathbf{s}}(t)=\frac{1}{V} \sum_{j=1}^{N} \frac{f(\mathbf{Z},)}{p(\mathbf{Z},)}+\epsilon, \quad \epsilon \simeq \frac{\sigma}{\sqrt{N}},
$$


where $N_{s}(t)$ is the total number of physical particles in the system at time $t$, and

$$
\sigma^{2}=\int\left(\frac{f(\mathbf{Z})}{p(\mathbf{Z})}-N_{s}\right)^{2} p(\mathbf{Z}) d \Gamma
$$

Since our Monte Carlo estimates are unbiased, we have

$$
\mathcal{E}\left(\frac{1}{N} \sum_{j=1}^{N} \frac{f\left(\mathbf{Z}_{j}\right)}{p\left(\mathbf{Z}_{j}\right)}\right)=N_{s},
$$

as it can be easily shown. However, the error term $\epsilon$ will not vanish in general. For the crude Monte Carlo method, with $p=1 / V$, we get

$$
\sigma^{2}=V \int\left(f-\frac{N_{s}}{V}\right)^{2} d \Gamma \neq 0 \text {. }
$$

Thus, the crude Monte Carlo will not conserve particles exactly, except when $f$ is a constant equal to the mean particle density in phase space. On the other hand, the importance sampling technique guarantees particle conservation trivially since each macro particle represents a fixed number of physical particles, and macro particle number is easily conserved numerically. This result also follows from the discussion above, with $p=f / N_{s}$, which leads to

$$
\sigma^{2}=\int\left(N_{s}-N_{s}\right)^{2} p d \Gamma=0
$$

identically.

The control variates- $\delta f$ method also fails to conserve particles exactly. To see this, we let $A=1$ in Eq. (17) to obtain:

$$
N_{s}(t)=N_{s o}+\frac{1}{N} \sum_{j=1}^{N} \frac{\delta f\left(\mathbf{Z}_{j}\right)}{p\left(\mathbf{Z}_{j}\right)}+\epsilon_{\delta f}, \quad \epsilon_{\delta f} \simeq \frac{\sigma_{\delta f}}{\sqrt{N}},
$$

where

$$
\sigma_{\delta f}^{2}=\int\left(\frac{\delta f}{p}-\left\langle\frac{\delta f}{p}\right\rangle\right)^{2} p d \Gamma .
$$

If $f_{0}$ is chosen such that $f_{0}=f(t=0)$, then

$$
\left\langle\frac{\delta f}{p}\right\rangle=\int\left(f-f_{0}\right) d \Gamma=0 .
$$


Then, in general,

$$
\sigma_{\delta f}^{2}=\int\left(\frac{\delta f}{p}\right)^{2} p d \Gamma \neq 0 .
$$

For instance, in the importance sampling version of the $\delta f$ method, with $p=f / N_{s}$, we have $\sigma_{\delta f}^{2}=N_{s}^{2} \overline{w_{j}^{2}}$, and $\epsilon \simeq N_{s} \sqrt{w_{j}^{2}} / N$, where $w_{j}$ is the particle weight. Obviously, $\epsilon \neq 0$ for $t>0$ in general, precluding exact particle conservation. However, we should note that, since all these methods use consistent estimates for the integrals in the sense discussed earlier, particle conservation becomes exact for all of them in the limit $N \rightarrow \infty$.

In the next section we turn to applications of some of these techniques to linear and nonlinear stability problems in nonneutral plasmas. First, standard electrostatic particle simulation techniques based on importance sampling method of Sec. II.B.2 are used to study stable and unstable diocotron modes. Next, low noise control variates- $\delta f$ method is applied to resonant diocotron modes, which have very small growth rates and cannot be studied efficiently with other particle simulation techniques.

\section{Applications to Nonneutral Plasmas}

A nonneutral plasma column of sufficiently low density immersed in a magnetic field can be confined for very long times while exhibiting a wealth of dynamical behavior. The good confinement characteristics are due to the presence of global conservation laws, ${ }^{15,16}$ while most of the interesting dynamics are the nonlinear manifestations of the "universal mode" of nonneutral plasmas, the ubiquitous diocotron mode. ${ }^{17,18}$ Like the Kelvin-Helmholtz instability of sheared fluid flows, the diocotron instability feeds on the potential energy of the sheared $\mathbf{E} \times \mathbf{B}$ drift velocity, which is an integral part of the equilibrium in these systems because of the space-charge generated $\mathbf{E}$ field and the confinening magnetic field. An extensive bibliography and a highly detailed theoretical discussion on the subject can be found in the treatise by Davidson. ${ }^{19}$ Some of the more recent experimental results using pure electron plasmas, 
with examples of long-lived coherent structures generated in such systems, are summarized by Driscoll and Fine. ${ }^{20}$

In this section, results from a number of numerical experiments, much in the same spirit as the pure electron plasma experiments performed at the University of California at San Diego referred to earlier, ${ }^{20}$ are presented. The geometry is that of a cylindrical pure electron plasma column of finite length, but with periodically identified ends, surrounded by a conducting cylinder. Electrostatic particle-in-cell (PIC) techniques, following either the full particle orbits, or only the guiding centers, are used to study the evolution of the electron plasma in a uniform axial magnetic field. The calculations are fully three dimensional, typically with a $64 \times 32 \times 8$ grid in $(r, \theta, z)$ directions, respectively; however, no axial modes are excited, and the preservation of translational symmetry during long nonlinear calculations is used as a check on numerical accuracy.

The first set of calculations involve stable diocotron modes with mode numbers $l=1$ and $l=2$; they are presented for comparison with the actual experiments at San Diego. ${ }^{20}$

\section{A. Stable $l=1$ and $l=2$ diocotron modes}

Stable $l=1$ diocotron modes are observed when, starting with a stable density profile with $\partial n / \partial r<0$ centered at the cylindrical axis, particles are subjected to a rigid displacement off axis of amplitude $d$. The resulting $l=1$ vortex rotates around the geometric axis with a frequency $\omega_{R} \simeq \omega_{D}$, where $\omega_{D}$ is the characieristic diocotron frequency of the original symmetric state given by $\omega_{D}=\omega_{p e}^{2} /\left(2 \Omega_{c e}\right)$, and $\omega_{p e}$ and $\Omega_{c e}$ are the electron plasma and cyclotron frequencies, respectively. ${ }^{19}$ The initial displaced state, which is not in equilibrium, and the stable vortex after approximately 70 rotations (somewhat less than $10^{5}$ rotations obse:ved in experiments!), without any apparent loss of confinement, are shown in Figs. la, and $b$, respectively. Figure $1 c$ shows contours of the electrostatic potential from which the $l=0$ components have been subtracted, giving essentially the potential in the rotating 
frame, which is in qualitative agreement with the analysis of Lund et al. ${ }^{21}$ The plasma crosssection in the poloidal plane and the rotation frequency are known to be affected by the proximity of the column to the conducting wall. In particular, experimental results indicate that the frequency shift is a quadratic function of the displacement of the column from the cylindrical axis. ${ }^{22}$ Our numerical results, shown in Fig. 2, also display a similar dependence, where we plot the relative change in the rotation frequency as a function of the displacement normalized to the radius of the conducting wall, $d / a$. A similar result has been found also by

Spencer and Mason in their numerical investigations of the culd-fluid equilibrium equation. ${ }^{23}$

A similar finite $l=2$ perturbation of an initially symmetric plasma column witb $\partial n / \partial r<$ 0 results in a stable $l=2$ vortex pattern, which again survives for a large number of rotations, as shown in Fig. 3. Figure 3a shows the initial, nonequilibrium state; Figs. 3b and 3c show beginning of the rotation and formation of two streamers at the tips, which eventually form a halo-like structure around the stable $l=2$ vortex, shown in Fig. 3d. Again, this result is quite similar to the experimentally observed $l=2$ vortices (See Fig. 6b in Ref. 20).

\section{B. Strongly unstable diocotron modes and vortex generation}

Strongly nonmonotonic density profiles are robustly unstable to diocotron modes ${ }^{17}$; the best example of this behavior is seen in hollow electron beams, which tend to break up into discrete vortices, again with very long confinement times (See, for example, Sec. 6.4 of Ref. 19). The stability of of these vortices is due to the presence of invariant integrals of motion, and they have been studied by many workers (e.g. Filippov and Yan'kov's4 and the references therein). An example of this behavior is shown in Fig. 4, where a hollow electron plasma column generates long-lived vortices. Fig. 4a shows the initial state with a small $l=8$ perturbation; the unstable diocotron mode, with a growth rate comparable to the $\mathbf{E} \times \mathbf{B}$ drift frequency, quickly breaks up the column into eight vortices, as shown in Fig. 4b. Subsequently, we see the formation of four vortex pairs (Fig. 4c), and eventually a 
slow merger in one of the pairs (Fig. 4d). The particles in the vortices have very complicated orbits: a particle in a given vortex drifts around the center of the vortex, while the vortex itself rotates around the center of mass of the vortex pair. Of course, the whole system of vortices continue to rotate around the cylindrical axis. For economic reasons, the calculation has not been continued long enough to determine the eventual fate of all vortices; indications are that, at least some of them, having been physically separated from others as a result of mergers, will survive for very long times.

\section{Resonant diocotron modes}

In addition to the modes discussed above, nonmonotonic profiles can also support "resonant diocotron moc's" with the resonance condition $\omega=\left(l / r_{s}\right) V_{E}\left(r_{s}\right)$, where $r_{s}$ is the radius of the resonance layer, and $V_{E}$ is the equilibrium $\mathbf{E} \times \mathbf{B}$ drift velocity. ${ }^{25}$ Here $\omega$, and $l$ are the mode frequency and the azimuthal (poloicial) mode number, respectively. These are negativeenergy waves that are weakly unstable when the resonance point falls in a layer of positive density gradient, $\partial n / \partial r>0$. Since the growth rate is typically very small, $\gamma \ll \omega_{r}$, where $\omega_{r}$ is the real part of the mode frequency, the usual simulation techniques (e.g. those based on importance sampling) are too noisy for resolution of these modes. The control variate- $\delta f$ method of Sec. II.B.3, on the other hand, is ideally suited for this problem, especially when the equilibrium profile is nearly monotonic. In that case, unlike the robustly unstable modes of the previous section that produce discrete vortices and completely change the equilibrium density profile, the resonant diocotron modes saturate at a relatively small amplitude, with only small modifications in the background equilibrium.

Linear growth and nonlinear saturation of resonant diocotron modes are examined for the density profile shown in Fig. 5 ; because of the small bump at $r \simeq 0.35$ in the initial profile (lengths are normalized to the conducting wall radius), resonant $l=2$ and $l=3$ modes are linearly unstable. Their evolution and nonlinear generation of other modes noodify the 
profile, reducing the bump and making the whole profile nearly monotonic, as it is also seen in Fig. 5. The inset in the same figure shows the saturated value of the difference $\delta n(r, \theta, t) \equiv n(r, \theta, t)-n_{0}(r, t)$, which is the quantity actually calculated by the control variate- $\delta f$ method. A slight broadening of the profile, lowering of the density bumps, and increase in the center density are clearly visible. We note that the change in the profile is small, with $\delta n / n_{0} \simeq 0.1$; use of the control variate- $\delta f$ method proves to be highly efficient because of this small relative change in the background density during the nonlinear phase. Figure 6 shows that the $\mathbf{E} \times \mathbf{B}$ drift freciuency profile $\left(\omega_{E}=V_{E} / r\right)$ also goes through a similar modification. Because of the lon oise nature of the $\delta f$ method, we are able to follow the linear phase of the modes thro gh over ten decades of exponentiation, as it is seen in Fig. 7, where time histories of the mode energies are plotted for the linearly unstable $l=2,3$ modes and some of the driven modes. For reasons not understood at this time, the growth rates for $l=2$ and $l=3$ are initially identical, $\gamma=2.14 \times 10^{-3}$ (in units where $\omega_{p e}=0.1$, although later on $l=3$ seems to dominate. The real part of the frequency is much higher, with $\omega_{2}=0.385$, and $\omega_{3}=0.569$; comparing this data with that of the equilibrium drift frequency in Fig. 6 shows that both modes are resonant at approximately $r=0.3$, in a region of positive density gradient, as expected from theory. ${ }^{26}$ Finally, Fig. 8 shows the real and imaginary parts of the eigenfunction $\phi_{l}(r)$ for $l=2,3$. We note that $\phi_{l}$ very quickly vanishes outside the plasma and is zero at the wall $(r=1)$, consistent with experimental observations for these unstable modes which show a zero wall signature. ${ }^{20}$

\section{Summary}

Using the well-known, but not often used, Monte Carlo viewpoint of particle simulations, we present a unified picture of various techniques developed over the years. Usual particle simulations are known to be based on the "importance sampling" Monte Carlo technique, where the computational particle are assumed to have the same distribution in phase space 
as the physical particles in the problem being investigated. This technique, although quite superior to the "crude Monte Carlo" technique where the computational particles are distributed uniformly in phase space, still suffers from noise problems that make it difficult and expensive to apply to certain problems. The $\delta f$ method based on "the control variates" Monte Carlo technique can overcome this noise problem and be quite useful in those applications where the time evolution of the distribution function does not take it too far from its original state so that $\delta f / f$ is always small.

Some of these techniques are applied to linear and nonlinear problems in nonneutral plasmas in the second part of the paper. A pure electron plasma column surrounded by a cylindrical conducting wall, with periodic end conditions, is used to study various stable and unstable diocotron modes. Long-lived $l=1$ and $l=2$ vortices, and others with higher mode numbers generated by unstable diocotron modes in hollow profiles, are studied and compared with experimental observations. Finally, low noise techniques are applied to "resonant diocotron modes," with growth rates much smaller than the characteristic rotation frequency in the problem. Unlike the unstable modes observed in hollow profiles that break up the column into discrete vortices, these modes saturate by more gentle modifications of the background equilibrium profile.

\section{Acknowledgments}

This work was supported by the U.S. Department of Energy under grant No. DE-FG0580ET-53088. Some of this work was performed while the author was a visiting professor at the National Institute for Fusion Science at Nagoya, Japan, as part of the Joint Institute for Fusion Theory exchange program. He sincerely thanks Profs. Sato, Okamoto, Watanabe, and other members of NIFS for their kind hospitality and many warm discussions during his stay. 


\section{References}

${ }^{1}$ Charles K. Birdsall and A. Bruce Langdon, Plasma Physics via Computer Simulations, McGraw-Hill (1985).

${ }^{2}$ R. W. Hockney and J. W. Eastwood, Computer Simulation Using Particles, McGrawHill (1981).

${ }^{3}$ J. U. Brackbill and B. I. Cohen, editors, Multiple Time Scales, Chapters 7-11, Academic Press (1985).

${ }^{4}$ J. M. Hammersley and D. C. Handscomb, Monte Carlo Methods, Chapman and Hall, London (1979).

${ }^{5}$ R. L. Morse, Methods in Computational Physics, Vol. 9, p. 213, Academic Press, New York (1970).

${ }^{6}$ D. V. Sivukhin, Reviews of Plasma Physics, Vol. 1, p. 96, Consultants Bureau, New York (1965).

'E. Goursat, A Course in Mathematical Analysis, Vol. II, pi. 2, p. 83, Dover, New York (1964).

${ }^{8}$ For a brief discussion of the terminology, the reader is referred to Chapter 2 of Hammersley and Handscom (Ref. 4).

${ }^{9}$ N. Metropolis, A. W. Rosenbluth, M. N. Rosenbluth, A. H. Teller, and E. Teller, J. Chem. Phys. 21, 1087 (1953).

${ }^{10}$ E. C. Fieller and H. O. Hartley, Biometrica 41, 494 (1954). 
${ }^{11} \mathrm{~T}$. Tajima and F. W. Perkins, Proceedings of the Sherwood Theory Conference, paper 2P9, Baltimore, MD (1983).

${ }^{12}$ M. Kotschenreuther, Bull. Am. Phys. Soc. 34, 2107 (1988).

${ }^{13}$ A. M. Dimits and W. W. Lee, J. Comp. Phys. 107, 309 (1993).

${ }^{14}$ S. E. Parker and W. W. Lee, Phys. Fluids B 5, 77 (1993).

${ }^{15}$ T. M. O'Neill, Phys. Fluids 23, 2216 (1980).

${ }^{16}$ Ronald C. Davidson, Phys. Fluids 27, 1804 (1984).

${ }^{17}$ R. H. Levy, Phys. Fluids 8, 1288 (1965).

${ }^{18}$ O. Buneman, R. H. Levy, and L. M. Lincon, J. Applied Phys. 37, 3203 (1966).

${ }^{19}$ Ronald C. Davidson, Physics of Nonneutral Plasmas, Addison-Wesley, Redwood City, CA (1990).

${ }^{20} \mathrm{C}$. F. Driscoll and K. S. Fine, Phys. Fluids B 2, 1359 (1990).

${ }^{21}$ Steven M. Lund, Jesus J. Ramos, and Ronald C. Davidson, Phys. Fluids B 5, 19 (1993).

${ }^{22}$ K. S. Fine, C. F. Driscoll, and J. H. Malmberg, Phys. Rev. Lett. 63, 2232 (1989).

${ }^{23}$ Ross L. Spencer and Grant W. Mason, Phys. Fluids B 5, 1738 (1993).

${ }^{24}$ D. V. Filippov and V V. Yan'kov, Soc. J. Plasma Phys. 12, 548 (1986).

${ }^{25}$ R. J. Briggs, J. D. Daugherty, and R. H. Levy, Phys. Fluids 13, 421 (1970).

${ }^{26}$ Ronald C. Davidson, Phys. Fluids 28, 1937 (1985). 


\section{Figure Captions}

1. Particle distribution and the electrostatic potential for an $l=1$ vortex.

a) The initial particle positions, b) positions after approximately 70 rotations, c) the potential contours without the $l=0$ component, thus showing the potential in a frame of reference cotating with the vortex.

2. Relative change in the rotation frequency as a function of the displacement of the center of mass from th? cylindrical axis for the $l=1$ vortex. Here $f_{0}$ is the diocotron frequency with no displacement. The so!id line is a best quadratic fit to the numerical data.

3. Particle positions for an $l=2$ vortex. a) The initial stationary state, which is not in equilibrium. b) and c) show formation of two streamers after the start of the rotation. d) The rotating equilibrium state, with a halo-like structure formed by the streamers around the vortex.

4. Formation of vortices by an unstable $l=8$ diocotron mode in a hollow profile. Notice the vortex pair formation in c), and the beginning of a merger in d).

5. A nonmonotonic profile with a gentle bump that is unstable to resonant diocotron modes. Both the initial contition and the saturated state are shown. The inset shows the difference between the two profiles in more detail.

6. Change in the $\mathbf{E} \times \mathbf{B}$ drift frequency profile induced by the nonresonant diocotron modes of Fig. 5.

7. Time histories of E-field energy for various modes for the nonlinear nonresonant diocotron calculation of Fig. 5. Only $l=2$ and $l=3$ are linearly unstable. The others appear to be driven modes.

8. Real and imaginary parts of the eigenfunction $\phi_{l}(r)$ for the nonresonant $l=2,3$ diocotron modes. Note that the potential vanishes outside the plasma. 


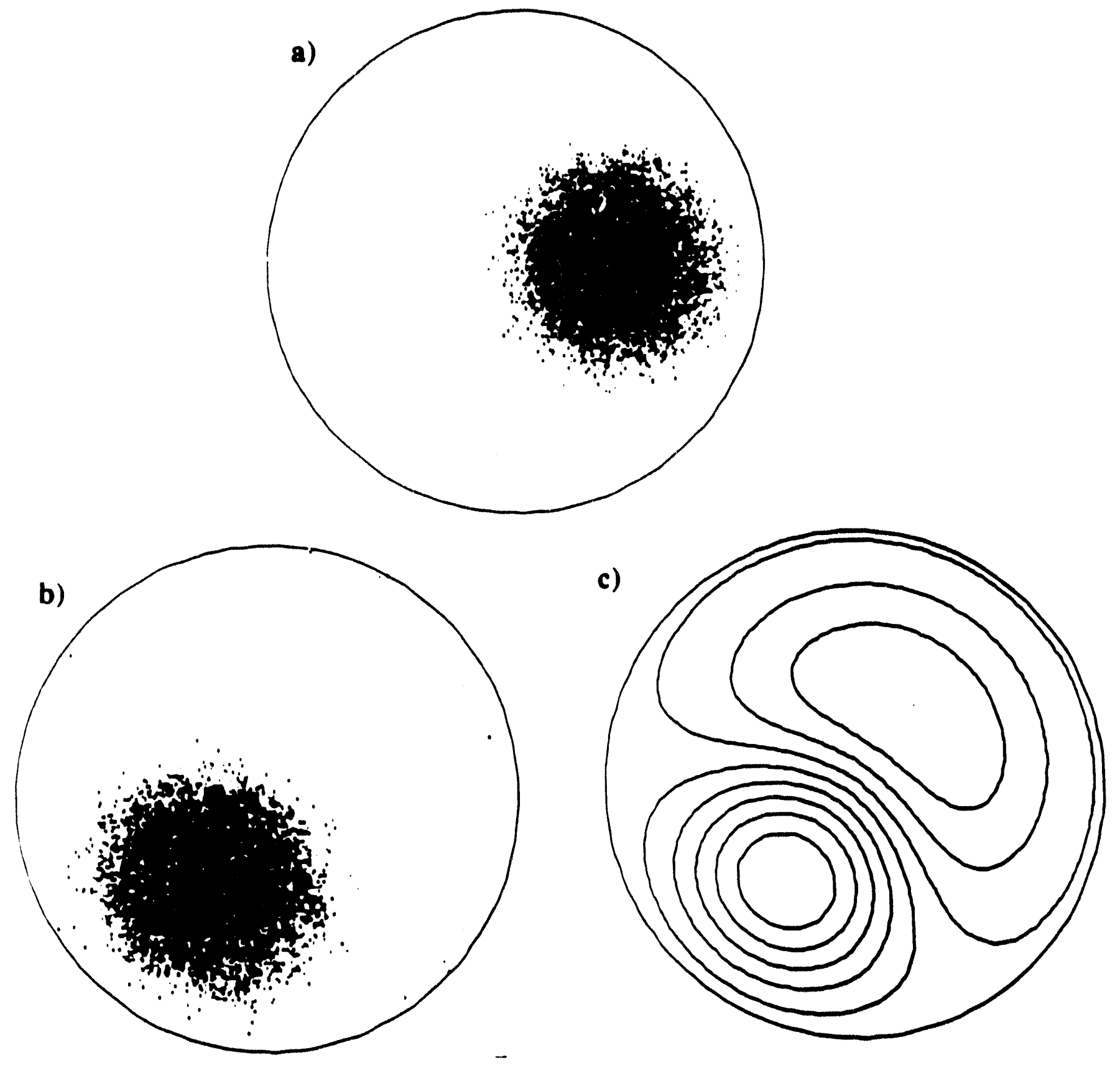

Fig. 1 
Relative Frequency Shift $(l=1)$



Fig. 2 


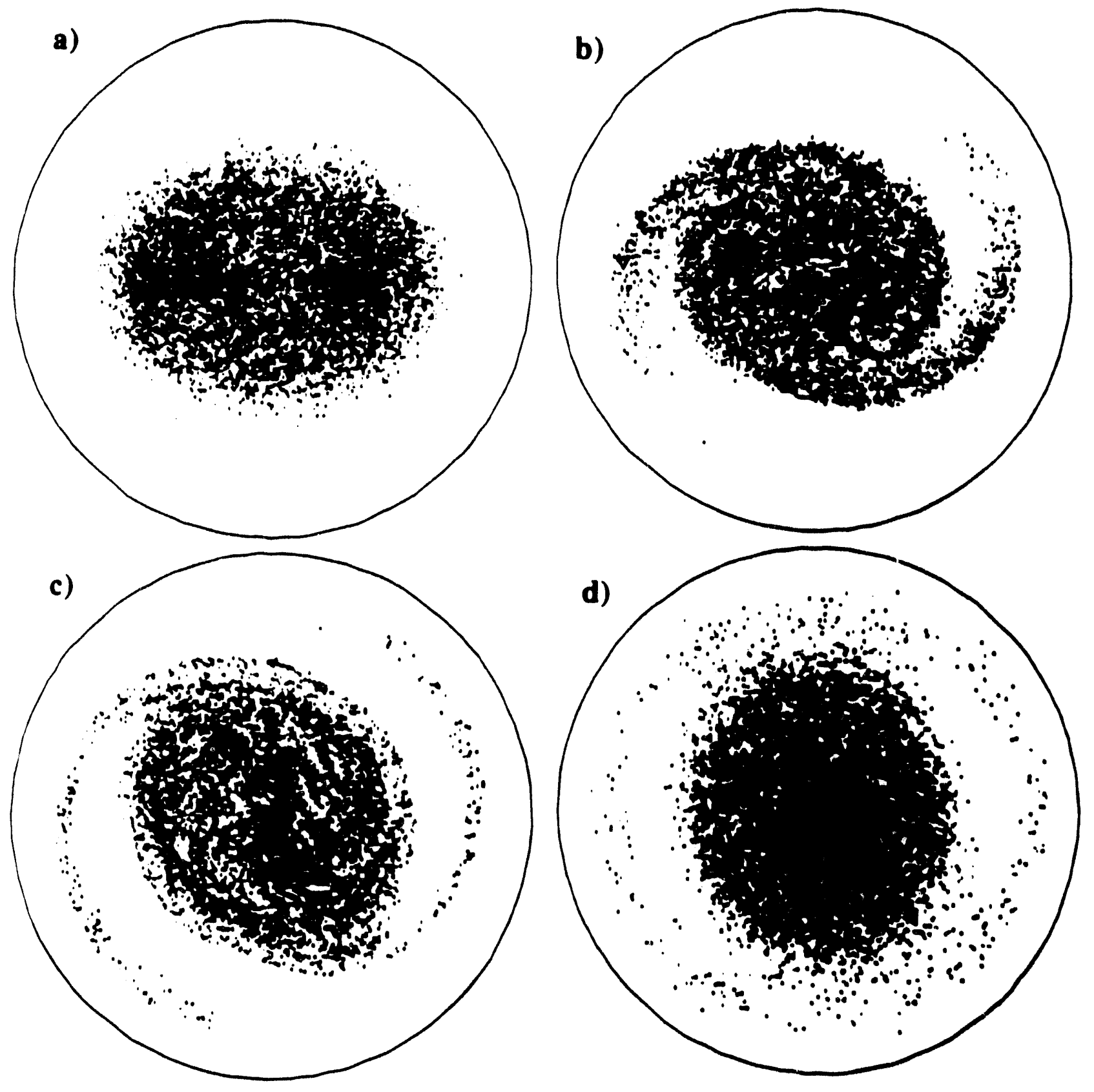

Fig. 3 

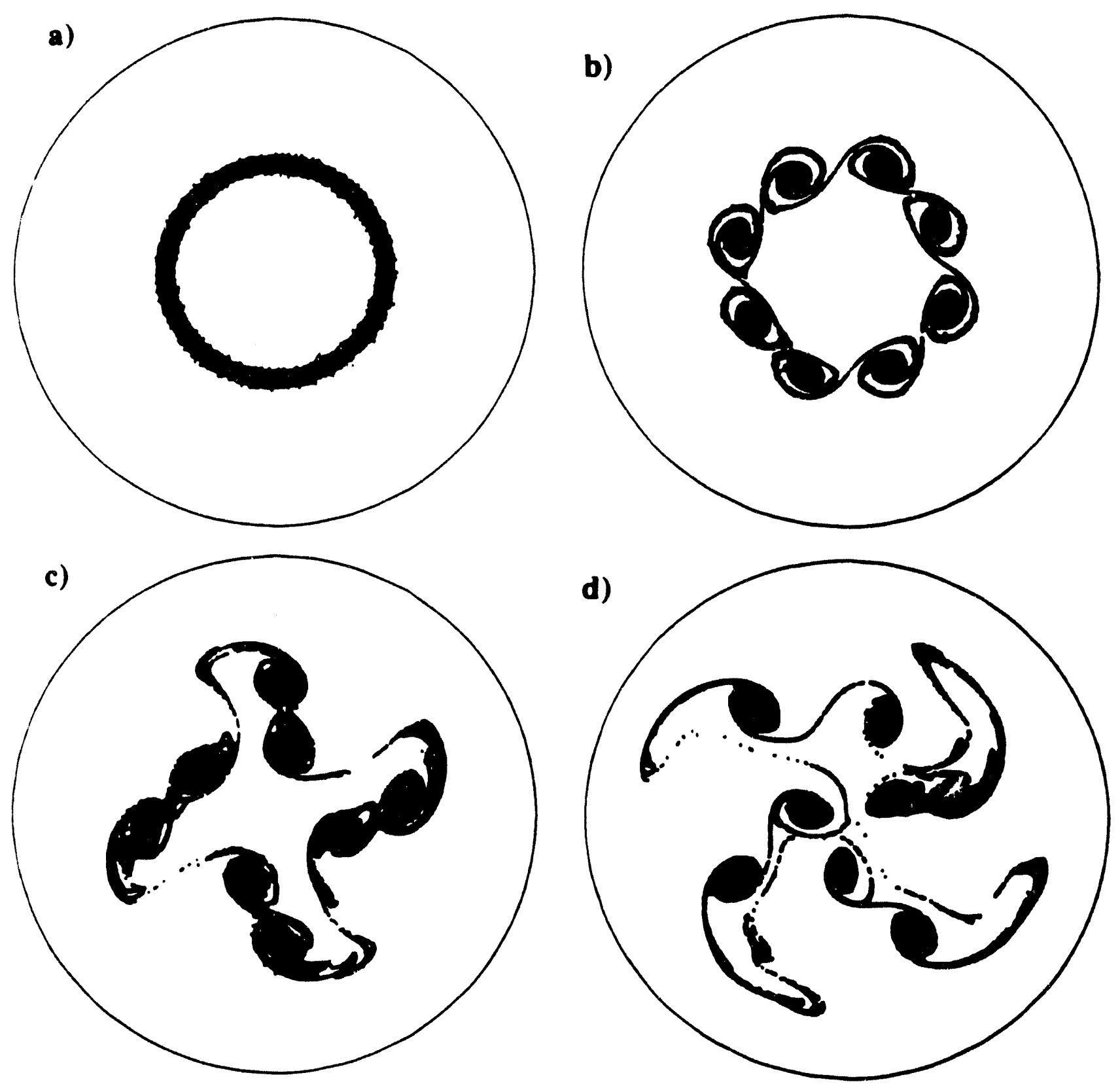

Fig. 4 
Change in Density Profile



Fig. 5 


\section{Change in Drift Frequency Profile}

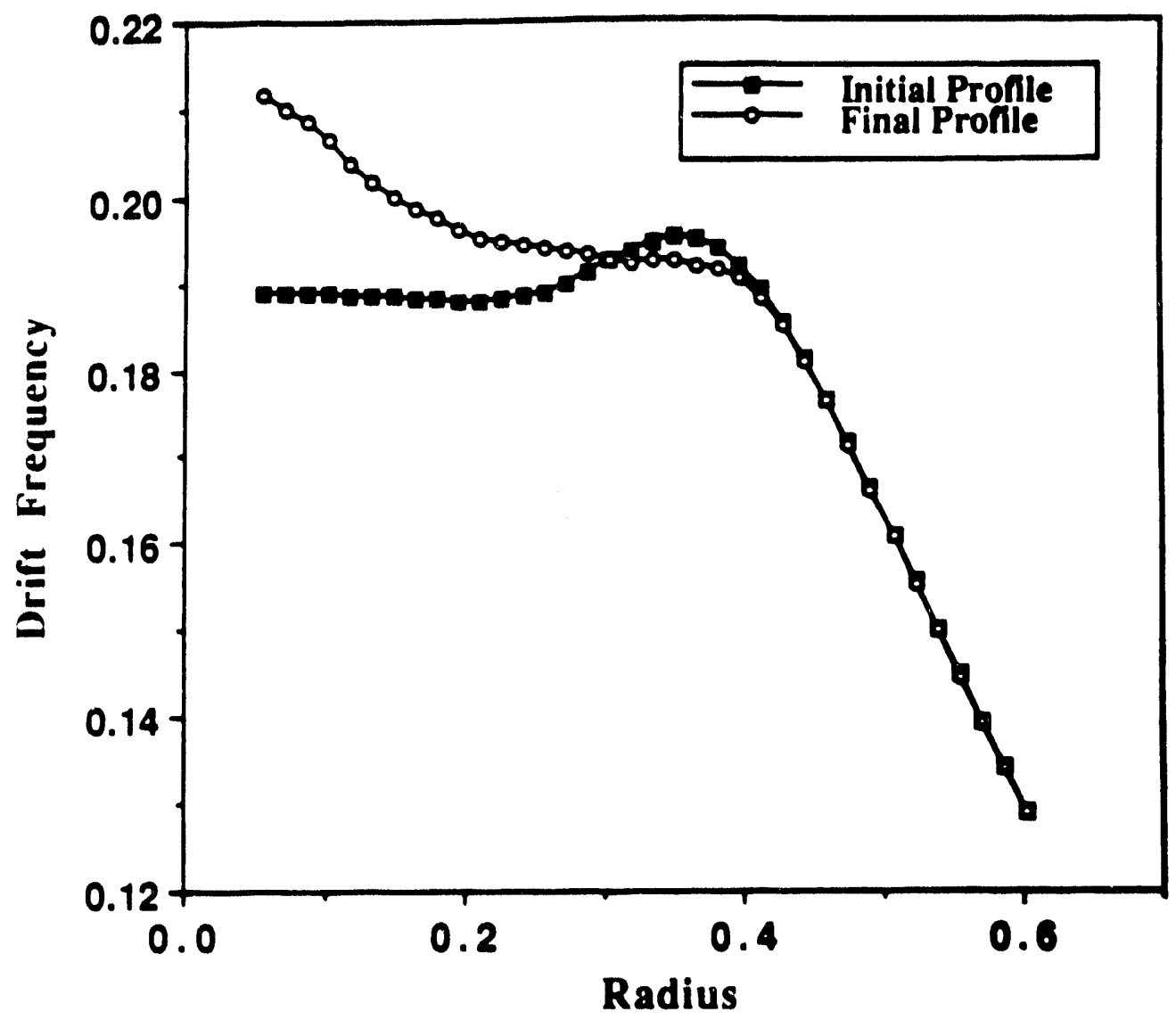

Fig. 6 


\section{E-Field Energy}

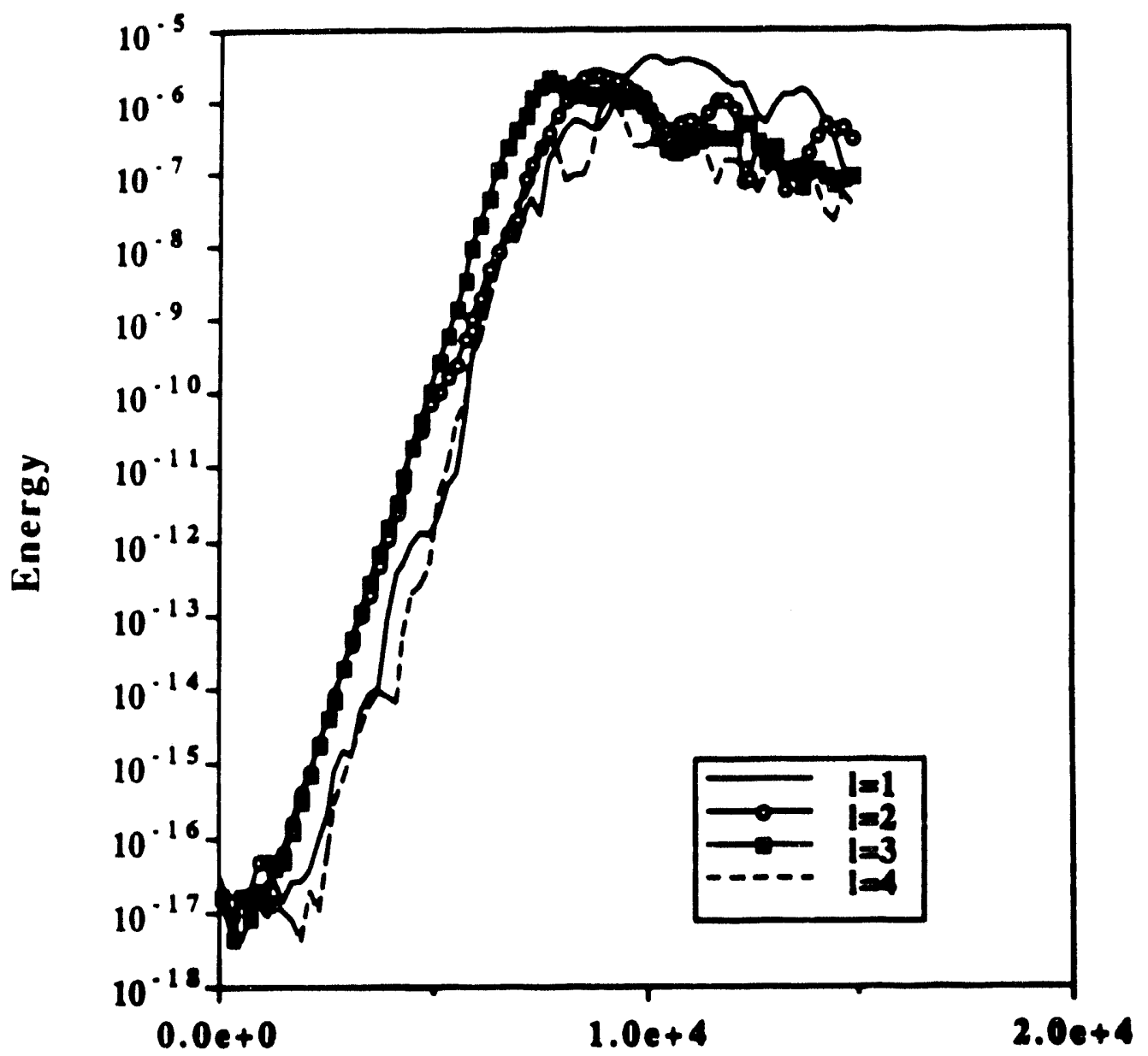

Time

Fig. 7 
Phi $(I=3)$



Phi (l=2)

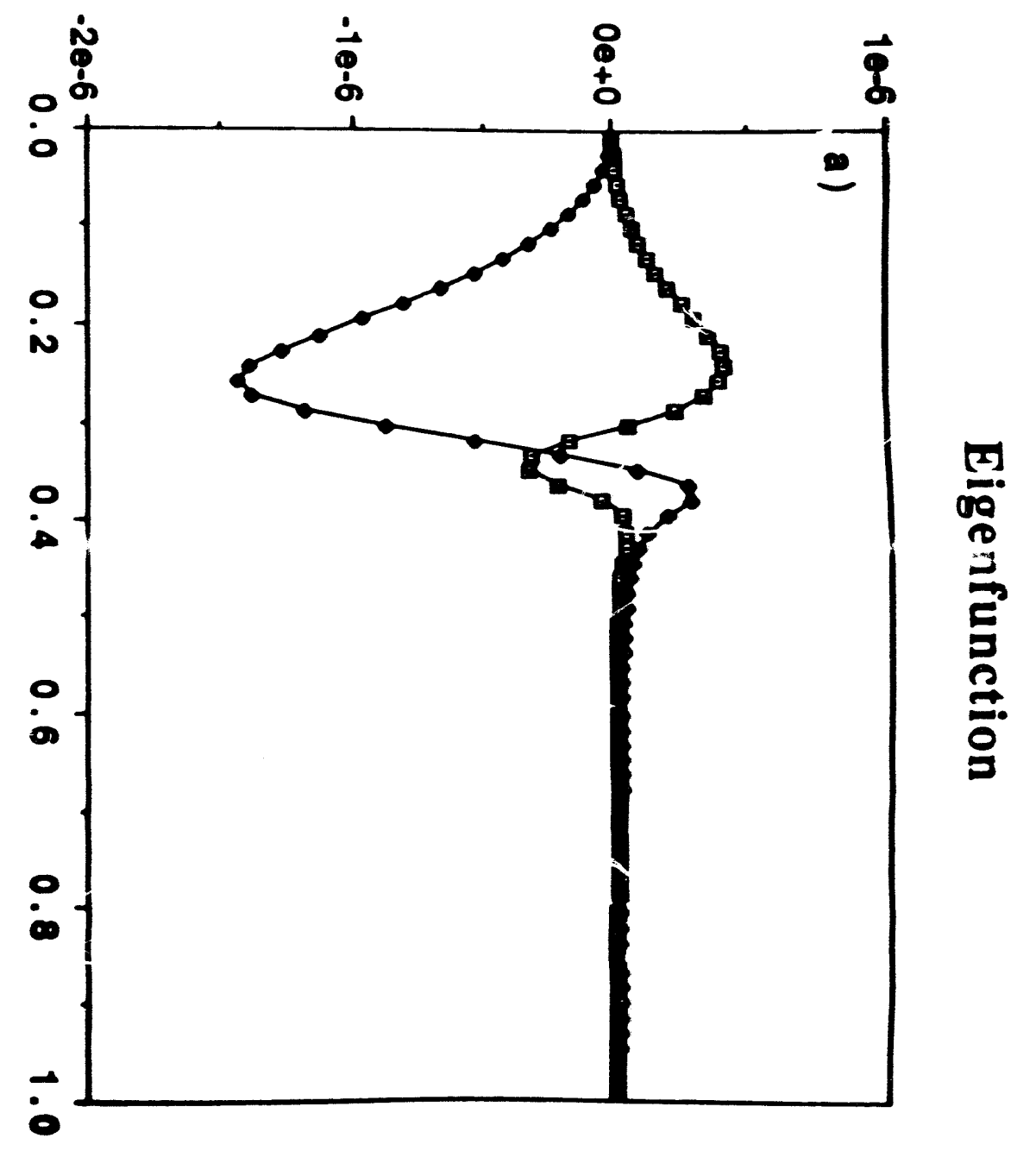



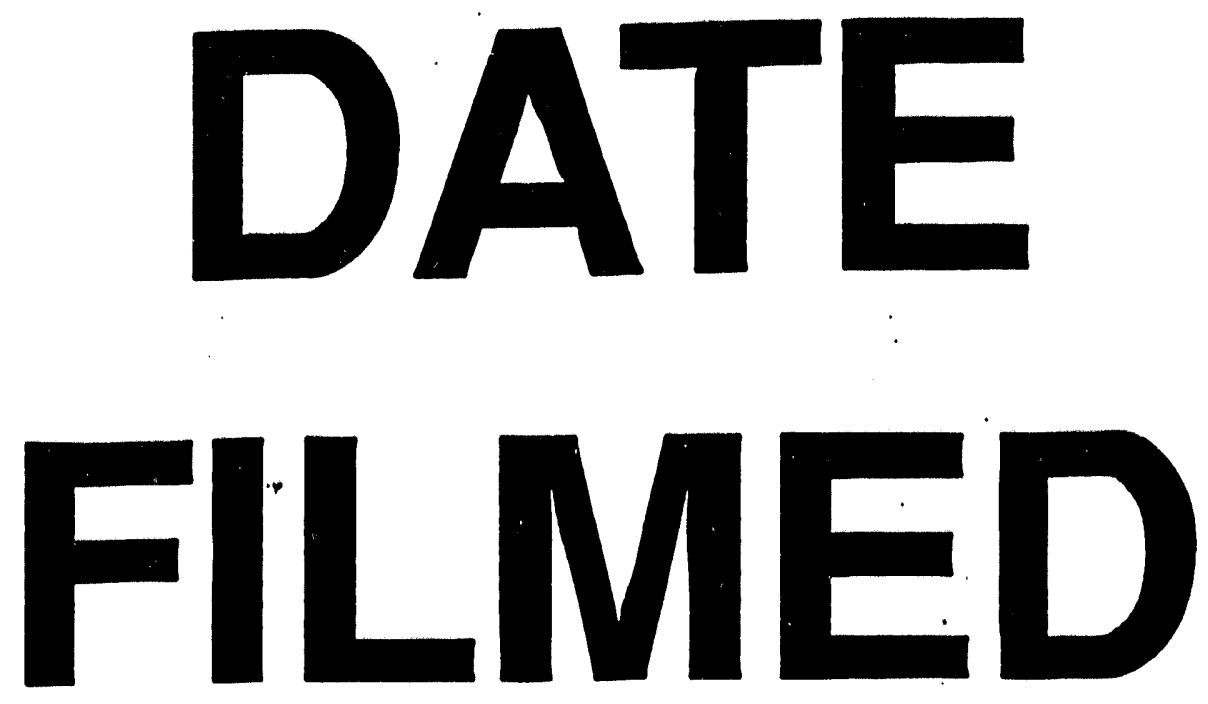

$11 / 23 / 93$
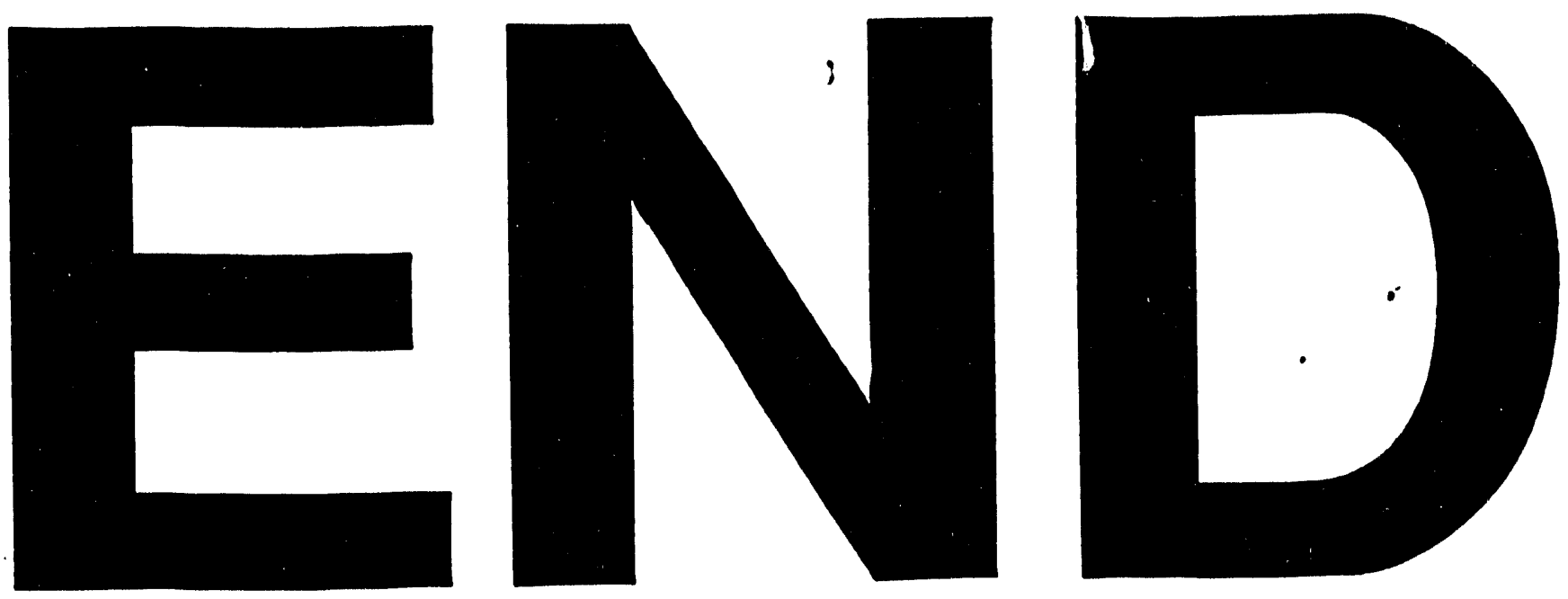
